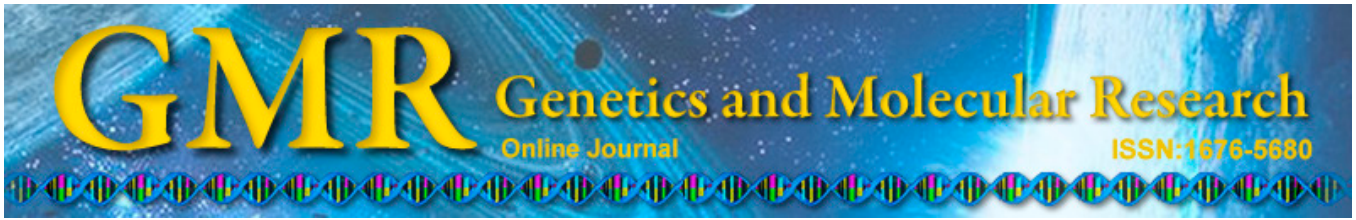

\title{
Boswellic acid attenuates asthma phenotype by downregulation of GATA3 via nhibition of PSTAT6
}

\author{
X. Zhou ${ }^{1 *}$, J.G. Cai ${ }^{2 *}$, W.W. Zhu' ${ }^{1}$, H.Y. Zhao ${ }^{1}$, K. Wang ${ }^{2}$ and X.F. Zhang ${ }^{2}$ \\ ${ }^{1}$ Department of Pediatrics, Jinan Central Hospital, Jinan, China \\ ${ }^{2}$ Department of Pediatrics, Shandong Provincial Qianfoshan Hospital, Jinan, \\ China \\ *These authors contributed equally to this study. \\ Corresponding author: J.G. Cai \\ E-mail: jgcai966@163.com
}

Genet. Mol. Res. 14 (3): 7463-7468 (2015)

Received October 14, 2014

Accepted January 29, 2015

Published July 3, 2015

DOI http://dx.doi.org/10.4238/2015.July.3.22

\begin{abstract}
To study the role of boswellic acid in reducing asthma phenotype severity and the relationship between the expression of pSTAT6 and GATA3, thirty-six mice were randomly divided into normal control group, asthma group, and boswellic acid group (treatment group). The asthma model was established through an intraperitoneal injection of sensitization liquid $(0.15 \mathrm{~mL}$ aluminum hydroxide gel at $88.67 \mathrm{mg} /$ $\mathrm{mL}$ and $0.05 \mathrm{mg}$ ovalbumin). pSTAT6 and GATA3 expression levels in peripheral blood were detected by reverse transcription-polymerase chain reaction and Western blot analysis. pSTAT6 and GATA3 gene expressions in the asthmatic group were significantly higher than in the normal control group; they were markedly lower in the treatment group than the asthma group, and there was no significant difference when compared with the normal control group. The pSTAT6 expressions in the asthma, control and treatment groups were $2.256 \pm 0.125,0.524$ \pm 0.210 , and $0.897 \pm 0.134$ at gray level, respectively. The GATA3 expressions in the asthma, control, and treatment groups were 3.521
\end{abstract}


$\pm 0.631,0.435 \pm 0.136$, and $0.743 \pm 0.149$ at gray level, respectively. pSTAT6 and GATA3 expression levels were similar in the treatment and control groups. GATA3 expression had a positive correlation with pSTAT6 expression. Boswellic acid may improve asthma symptoms by inhibiting pSTAT6 expression, which consequently reduces GATA3 expression.

Key words: Asthma; Boswellic acid; pSTAT6; GATA3

\section{INTRODUCTION}

Bronchial asthma is a common allergic disease caused by immune deficiency. Immune system has a predominant Th2-type response to bronchial asthma, which involves a variety of inflammatory cells. Its pathogenesis is closely linked to the changing ratio of Th1/Th 2 cytokines and mainly depends on increasing Th 2 cell count. Therefore, effectively controlling the increase of Th2-type cells or facilitating cell differentiation to the Th1 cell type to inhibit cytokine generation is one of the popular areas in the investigation of therepeutic agents for asthma (Hwang et al., 2011; Han et al., 2012; Shin et al., 2013). A previous study found that the transcription factor GATA, from the zinc finger protein family, plays an important role in the regulation of T-cell development into Th2 cells. It is upregulated during asthma pathogenesis and participates in a variety of inflammatory reaction processes. The pSTAT6 sequence has a high level of homology with others in the STAT family. pSTAT6 expression also increases during the pathogenesis of asthma, which indicates that pSTAT6 and GATA3 have different regulatory roles in this process (Mandal et al., 2010; Poritz et al., 2010; McCallister et al., 2013).

Boswellic acid, from frankincense, is a drug that can arrest pain by regulating respiratory rhythm and promoting blood circulation. It has significant therapeutic effects in various diseases, such as oral cavity ulcer, heart pain, and cancer. Additionally, an antitumor effect has also been reported. Clinically, it is one of the main drugs used in the treatment of asthma. Several studies have hypothesized that boswellic acid functions by affecting inflammatory factors; however, the exact mechanism is not yet clear (Ticconi et al., 2013; Bezerra et al., 2014). Our study investigated the role of boswellic acid in combination with the asthma-associated transcription factors pSTAT6 and GATA3 with a view to discover its mechanism of action in asthma.

\section{MATERIAL AND METHODS}

\section{Animal information}

SPF grade BALB/c male mice (6 to 7 weeks old) were bred in an experimental animal center in Jinan, Shandong. The mice weighed 18 to $22 \mathrm{~g}$ and were randomly divided into normal control, asthma, and boswellic acid groups with 12 mice in each group. Mice in the asthma group were 6.5 weeks old, and the average weight was $20.12 \mathrm{~g}$; mice in the control group were 6.3 weeks old with an average weight of $21.12 \mathrm{~g}$; mice in the treatment group were 6.7 weeks old and the average weight was $20.32 \mathrm{~g}$. No statistical differences were observed in the animals' age and weight in each group. All animals were raised and handled in strict accordance with the Animal Ethical Standard; this study was approved by the Ethics Committee of the Experimental Animal Center in Jinan, Shandong Province. 


\section{Reagents}

The RNA extraction kit was bought from TaKaRa Company (Dalian, China), and the RT-PCR kit was obtained from Promega (Madison, WI, USA). Reagents used for the Western blot were obtained from Beyotime Company. Primary antibodies for pSTAT6, GATA3, and $\beta$-actin were obtained from Xinlebio Company (Shanghai, China). The primers used in the study are shown in Table 1.

Table 1. Primers used in the study.
\begin{tabular}{llc}
\hline Primer name & Sequence (5'-3') & Product length (bp) \\
\hline pSTAT6 F & ACAGAGTCTGTCACCGAAGAGAAGT & 608 \\
pSTAT6 R & CGGATGACATGGGCAATGGTGATGC & 704 \\
GATA3 F & ACCCACCACGGGAGCCAGGTATGCC & \\
GATA3 R & CCTTGCTGCCGACAGCCTTCGCTTG & 281 \\
$\beta$-actin F & TCAGGTCATCACTATCGGCAA & \\
$\beta$-actin R & AAAGAAAGGTTGTAAACGCA & \\
\hline
\end{tabular}

\section{Methods}

\section{Asthma modeling}

To establish the asthma model (Poritz et al., 2010), $0.2 \mathrm{~mL}$ sensitization liquid [containing $0.15 \mathrm{~mL}$ aluminum hydroxide gel at $88.67 \mathrm{mg} / \mathrm{mL}$ and $0.05 \mathrm{mg}$ ovalbumin (OVA)] was injected intraperitoneally at 0,7 , and 14 days. After 3 weeks, the animals were maintained in airtight atomization inhalation cases. Saline $(200 \mu \mathrm{L})$ was injected intraperitoneally, and after $30 \mathrm{~min}$, the mice were excited with 1\% OVA atomization for $30 \mathrm{~min}$ to trigger asthma. The control group were administered normal saline. Boswellic acid $(100 \mathrm{mg} / \mathrm{kg})$ was injected intraperitoneally before atomization excitation in the treatment group. The animals were sampled $24 \mathrm{~h}$ after the last excitation.

\section{Reverse transcription polymerase chain reaction (RT-PCR)}

The peripheral blood was collected, and total RNA was extracted by using Trizol. The extracted RNA was reverse transcribed into cDNA by using PCR. The cycling conditions consisted of an initial single cycle of $5 \mathrm{~min}$ at $94^{\circ} \mathrm{C}$, followed by 35 cycles of $30 \mathrm{~s}$ at $94^{\circ} \mathrm{C}$, $25 \mathrm{~s}$ at $56^{\circ} \mathrm{C}$, and $60 \mathrm{~s}$ at $72^{\circ} \mathrm{C}$. The PCR products were subsequently detected by agarose gel electrophoresis.

\section{Western blot analysis}

Peripheral blood was collected and homogenized with lysis buffer. Total protein was separated by using denaturing $10 \%$ sodium dodecyl sulfate-polyacrylamide gel electrophoresis. The proteins were detected by using simple Western assay. The antibody dilutions in the ratio of 1:200 were used for p-STAT6, GATA3, and $\beta$-actin. Protein levels were normalized to $\beta$-actin and differences were determined. 


\section{RESULTS}

\section{mRNA expression of pSTAT6 and GATA3}

The mRNA expression of pSTAT6 and GATA3 is shown in Figure 1A and B. pSTAT6 and GATA 3 mRNA expressions in the asthma group were significantly higher than those in the control and treatment groups. In particular, changes in the GATA3 expression were positively correlated with pSTAT6 expression.

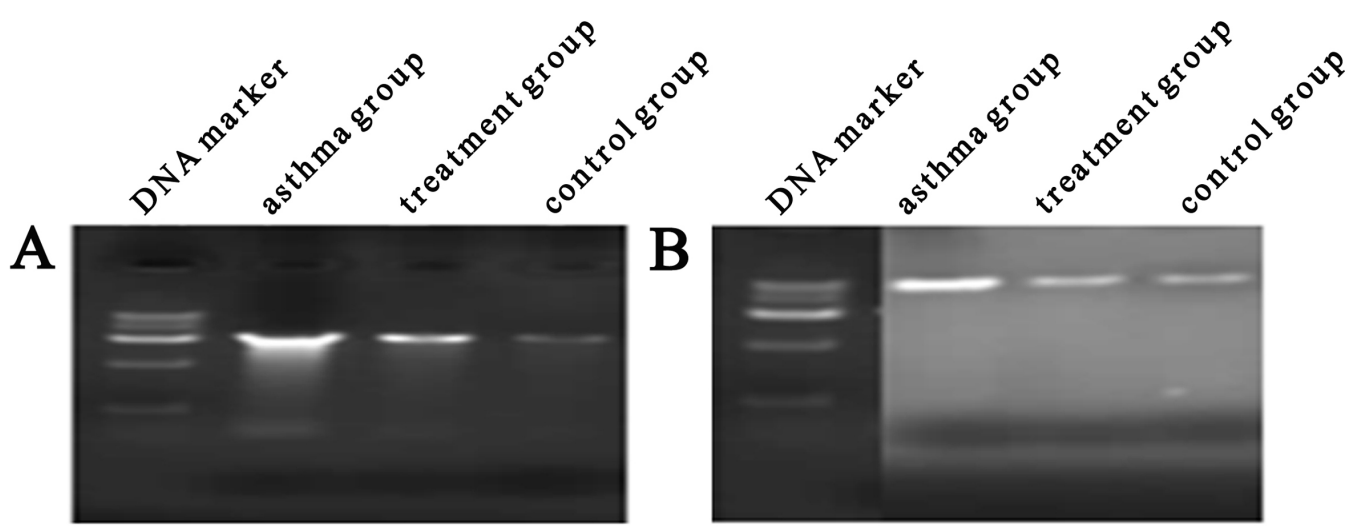

Figure 1. Protein expression of pSTAT6 and GATA3. A. pSTAT6 mRNA expression; B. GATA3 mRNA expression.

\section{Protein expression of pSTAT6 and GATA3}

Protein expression of pSTAT6 and GATA3 is shown in Figure 2, and the protein levels were calculated from the corresponding bands and listed in Table 2. pSTAT6 and GATA3 protein expression in the asthma group was significantly higher than in the control and treatment groups. Changes in GATA3 expression were influenced by pSTAT6, and hence, GATA3 was shown to be positively correlated with pSTAT6.

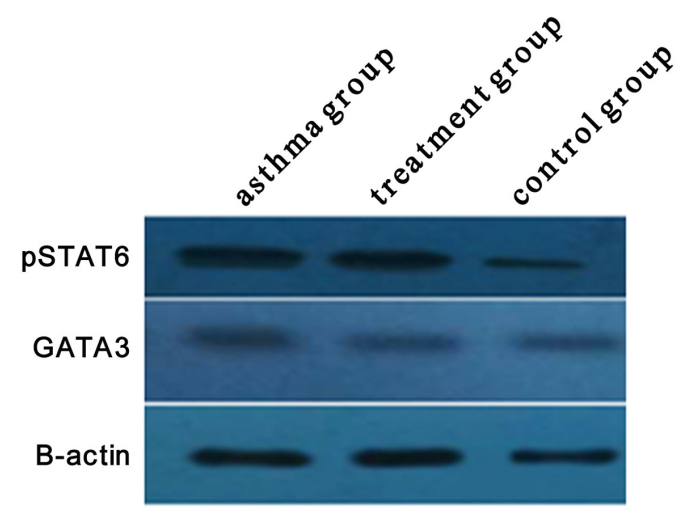

Figure 2. pSTAT6 and GATA3 protein expression. 
Table 2. pSTAT6 and GATA3 expression analysis.

\begin{tabular}{lcc}
\hline Group & pSTAT6 & GATA3 \\
\hline Asthma group & $2.256 \pm 0.125^{*}$ & $3.521 \pm 0.631^{*}$ \\
Control group & $0.524 \pm 0.210$ & $0.435 \pm 0.136$ \\
Treatment group & $0.897 \pm 0.134$ & $0.743 \pm 0.149$ \\
$t$-value & 1.256 & 0.536 \\
\hline
\end{tabular}

*Compared with control group and treatment group, $\mathrm{P}<0.05$.

\section{DISCUSSION}

Asthma is one of the most commonly occurring bronchial diseases. It is caused by bronchial convulsion and inflammation, resulting in restriction of airflow. It seriously affects the lives of patients (Hwang et al., 2011; Han et al., 2012; Shin et al., 2013). The main clinical manifestations include airway subepithelial fibrosis, basement membrane thickening, and smooth muscle hyperplasia that can cause airway narrowing and sustained hyper-responsiveness, which result in irreversible airflow obstruction. Currently, research in asthma pathogenesis focuses on the impact of inflammation such as changes in eosinophil and lymphocyte concentrations. Clinical treatment mainly depends on the use of on anti-inflammatory drugs such as curcumin, which has an effective anti-inflammatory and antioxidative function to relieve asthma and aid in lung reconstruction and recovery. Other drugs such as glucocorticoid hormones are also effective.

The transcription factor GATA3 belongs to the GATA zinc finger family of proteins that plays a key role in regulating the Th2 activation process. Several studies have confirmed that it was overexpressed in the pathogenesis of asthma. pSTAT6 is one of STAT6 gene products, and its DNA sequence is highly homologous with the others in the same family. A transcriptional activity area was found at STAT6's carboxyl end. Its phosphorylation plays a key role in interleukin-4-induced STAT6 activation. In addition, pSTAT6 expression also appears to increase in the pathogenesis of asthma. All of these indicate that pSTAT6 and GATA3 have different pathological functions (Mandal et al., 2010; Poritz et al., 2010; McCallister et al., 2013).

In our study, we selected boswellic acid for its anti-inflammatory and antioxidant effects, and because it is an effective drug in the clinical treatment of asthma. Several studies have shown that boswellic acid can significantly reduce the incidences of asthma (Mandal et al., 2010; Ticconi et al., 2013; Bezerra et al., 2014; Fatima and Osunkoya, 2014) . It was found that not only can boswellic acid be used to treat asthma but also exert antitumor effects. Furthermore, it was effective in treating rheumatism, rheumatoid arthritis, colitis, and inflammatory reactions (Yao et al., 2011; Dubovsky et al., 2011; Souza et al., 2012; Li et al., 2013; Menet et al., 2013; Chakilam et al., 2013). Due to the relationship between asthma and inflammation, we found it valuable to investigate the expression of transcription factors associated with the inflammatory reaction to clarify the mechanism of action of boswellic acid.

Thirty-six SPF BALB/c mice were randomly divided into three groups: normal control, asthma, and boswellic acid treatment. The asthma model was established through sensitization and stimulation by aluminum hydroxide and OVA, respectively. RT-PCR and Western blot analyses were used to detect the expressions of pSTAT6 and GATA3 in peripheral blood. Boswellic acid was found to be effective in improving the phenotype of asthma in mice; the expressions of both pSTAT6 and GATA3 decreased. In particular, GATA3 expression was positively correlated with pSTAT6, which indicated a certain regulatory relationship between 
pSTAT6 and GATA3. As we know, pSTAT6 can be regulated by many factors in the body and change in pSTAT6 correlates with many diseases (Batra et al., 2004; Thunberg et al., 2010; Chen et al., 2013; Yao et al., 2013; Irvin et al., 2014). Our present results prove that after boswellic acid treatment, GATA3 is downregulated following a decrease in pSTAT6, which indicates that boswellic acid may improve the asthma phenotype by inhibiting pSTAT6 and thus influencing GATA3 expression (Tachdjian et al., 2010).

\section{REFERENCE}

Batra V, Musani AI, Hastie AT, Khurana S, et al. (2004). Bronchoalveolar lavage fluid concentrations of transforming growth factor (TGF)-beta1, TGF-beta2, interleukin (IL)-4 and IL-13 after segmental allergen challenge and their effects on alpha-smooth muscle actin and collagen III synthesis by primary human lung fibroblasts. Clin. Exp. Allergy 34: 437-444.

Bezerra SM, Lotan TL, Faraj SF, Karram S, et al. (2014). GATA3 expression in small cell carcinoma of bladder and prostate and its potential role in determining primary tumor origin. Hum Pathol. 45: 1682-1687.

Chakilam S, Gandesiri M, Rau TT, Agaimy A, et al. (2013). Death-associated protein kinase controls STAT3 activity in intestinal epithelial cells. Am. J. Pathol. 182: 1005-1020.

Chen Z, Wang S, Erekosima N, Li Y, et al. (2013). IL-4 confers resistance to IL-27-mediated suppression on CD4+ T cells by impairing signal transducer and activator of transcription 1 signaling. J. Allergy Clin. Immunol. 132: $912-$ 921 e1-5.

Dubovsky JA, Powers JJ, Gao Y, Mariusso LF, et al. (2011). Epigenetic repolarization of T lymphocytes from chronic lymphocytic leukemia patients using 5-aza-2'-deoxycytidine. Leuk. Res. 35: 1193-1199.

Fatima N and Osunkoya AO (2014). GATA3 expression in sarcomatoid urothelial carcinoma of the bladder. Hum. Pathol. 45: $1625-1629$

Han YS, Kim MS and Hwang JK (2012). Macelignan inhibits histamine release and inflammatory mediator production in activated rat basophilic leukemia mast cells. Inflammation 35: 1723-1731.

Hwang SS, Kim K and Lee GR (2011). Defective GATA-3 expression in Th2 LCR-deficient mice. Biochem. Biophys. Res. Commun. 410: 866-871.

Irvin C, Zafar I, Good J, Rollins D, et al. (2014). Increased frequency of dual-positive T2/T17 cells in bronchoalveolar lavage fluid characterizes a population of patients with severe asthma. J. Allergy Clin. Immunol. 134: 1175-1186 e7.

Li N, Liu YH, Li SL, Fu CY, et al. (2013). Protective role of synthetic oligodeoxynucleotides expressing immunosuppressive TTAGGG motifs in concanavalin A-induced hepatitis. Immunol. Lett. 151: 54-60.

Mandal D, Fu P and Levine AD (2010). REDOX regulation of IL-13 signaling in intestinal epithelial cells: usage of alternate pathways mediates distinct gene expression patterns. Cell Signal. 22: 1485-1494.

McCallister JW, Holbrook JT, Wei CY, Parsons JP, et al. (2013). Sex differences in asthma symptom profiles and control in the American Lung Association Asthma Clinical Research Centers. Respir. Med. 107: 1491-1500.

Menet CJ, Rompaey LV and Geney R (2013). Advances in the discovery of selective JAK inhibitors. Prog. Med. Chem. 52: $153-223$

Poritz LS, Zhang WJ, Thompson J, Boyer M, et al. (2010). Impaired IL-4 phosphorylation of STAT6 in EBV transformed B-cells. J. Surg. Res. 162: 290-298.

Shin K, Chung HC, Kim DU, Hwang JK, et al. (2013). Macelignan attenuated allergic lung inflammation and airway hyper-responsiveness in murine experimental asthma. Life Sci. 92: 1093-1099.

Souza PP, Palmqvist P, Lundberg P, Lundgren I, et al. (2012). Interleukin-4 and interleukin-13 inhibit the expression of leukemia inhibitory factor and interleukin-11 in fibroblasts. Mol. Immunol. 49: 601-610.

Tachdjian R, Al Khatib S, Schwinglshackl A, Kim HS, et al. (2010). In vivo regulation of the allergic response by the IL-4 receptor alpha chain immunoreceptor tyrosine-based inhibitory motif. J. Allergy Clin. Immunol. 125: 1128-1136 e8.

Thunberg S, Gafvelin G, Nord M, Grönneberg R, et al. (2010). Allergen provocation increases TH2-cytokines and FOXP3 expression in the asthmatic lung. Allergy 65: 311-318.

Ticconi C, Pietropolli A and Piccione E (2013). Estrogen replacement therapy and asthma. Pulm. Pharmacol. Ther. 26: 617-623.

Yao W, Tepper R and Kaplan MH (2011). Predisposition to the development of IL-9-secreting T cells in atopic infants. $J$. Allergy Clin. Immunol. 128: 1357-1360 e5.

Yao W, Zhang Y, Jabeen R, Nguyen ET, et al. (2013). Interleukin-9 is required for allergic airway inflammation mediated by the cytokine TSLP. Immunity 38: 360-372 